\title{
Diffusiophoretic mobility of charged porous spheres in electrolyte gradients
}

\author{
Yeu K. Wei and Huan J. Keh* \\ Department of Chemical Engineering, National Taiwan University, Taipei 106-17, Taiwan, Republic of China
}

Received 3 April 2003; accepted 1 August 2003

\begin{abstract}
The diffusiophoretic motion of a polyelectrolyte molecule or charged floc in an unbounded solution of a symmetrically charged electrolyte with a uniform prescribed concentration gradient is analytically studied. The model used for the particle is a porous sphere in which the density of the hydrodynamic frictional segments, and therefore also that of the fixed charges, is constant. The electrokinetic equations which govern the electrostatic potential profile, the ionic concentration distributions (or electrochemical potential energies), and the fluid velocity field inside and outside the porous particle are linearized by assuming that the system is only slightly distorted from equilibrium. Using a regular perturbation method, these linearized equations are solved for a charged porous sphere with the density of the fixed charges as the small perturbation parameter. An analytical expression for the diffusiophoretic mobility of the charged porous sphere in closed form is obtained from a balance between its electrostatic and hydrodynamic forces. This expression, which is correct to the second order of the fixed charge density of the particle, is valid for arbitrary values of $\kappa a$ and $\lambda a$, where $\kappa$ is the reciprocal of the Debye screening length, $\lambda$ is the reciprocal of the length characterizing the extent of flow penetration inside the particle, and $a$ is the particle radius. Our result to the first order of the fixed charge density agrees with the corresponding solution for the electrophoretic mobility obtained in the literature. In general, the diffusiophoretic mobility of a porous particle becomes greater as the hindrance to the diffusive transport of the solute species inside the particle is more significant.
\end{abstract}

(c) 2003 Elsevier Inc. All rights reserved.

Keywords: Diffusiophoresis; Polyelectrolyte particles; Charged porous spheres

\section{Introduction}

A colloidal particle can be driven to move by the application of a nonuniform solute concentration field that interacts with the surface of the particle. This phenomenon, known as diffusiophoresis [1-3], has been demonstrated experimentally for both charged [4] and uncharged [5] solutes. Diffusiophoresis is of practical importance in some applications to particle analysis or separation and in certain latex-particle coating processes $[1,6]$. In a solution of nonionic solute, the solute molecules interact with the particle through the van der Waals/dipole forces. For charged particles in an electrolyte solution, the particle-solute interaction is electrostatic in nature and its range is the Debye screening length $\kappa^{-1}$. Particles with zeta potentials of order $k T / e(\sim 25 \mathrm{mV} ; e$ is the elementary electric charge, $k$ is Boltzmann's constant,

\footnotetext{
* Corresponding author.

E-mail address: huan@ntu.edu.tw (H.J. Keh).
}

and $T$ is the absolute temperature) in electrolyte gradients of order $1 \mathrm{M} / \mathrm{cm}$ will move by diffusiophoresis at speeds of several microns per second.

Using the classical model of the diffuse electric double layer, Prieve [7] derived a formula for the diffusiophoretic velocity of a nonconducting particle of arbitrary shape in a solution of a symmetrically charged electrolyte with a constant concentration gradient for arbitrary zeta potential $(\zeta)$ of the particle in the limit of thin double layer ( $\kappa a \rightarrow \infty$, where $a$ is the linear dimension of the particle). This formula agrees with that previously deduced by a more intuitive method [1]. Using a method of matched asymptotic expansions with $(\kappa a)^{-1}$ as the small parameter, Prieve et al. [2] evaluated the effect of particle curvature on the diffusiophoretic mobility of a spherical particle accurate to $\mathrm{O}\left[(\kappa a)^{-1}\right]$. When the double-layer distortion from equilibrium was taken as a small perturbation, Prieve and Roman [8] obtained a numerical solution for the diffusiophoresis of a dielectric sphere in concentration gradients of 1:1 electrolytes $(\mathrm{KCl}$ or $\mathrm{NaCl})$, which was applicable to a 
broad range of $\zeta$ and $\kappa a$. Later, analytical expressions for the diffusiophoretic velocity of a dielectric sphere with a "thin but polarized" double layer were also derived $[9,10]$. Recently, we have obtained analytical formulas in closed forms for the diffusiophoretic mobility of a colloidal sphere [11] and cylinder [12] in symmetric electrolytes at low surface charge density $\sigma$ (valid for $\zeta$ up to $50 \mathrm{mV}$ ) and arbitrary $\kappa a$. All results of the above investigations show that the diffusiophoretic mobility of the particle in general decreases with the increase of $(\kappa a)^{-1}$. Also, the particle can reverse the direction of its migration when the value of $|\zeta|,(\kappa a)^{-1}$, or the valence of the electrolyte increases.

A theoretical study of the electrokinetic phenomena of charged porous particles was first made by Hermans and $\mathrm{Fu}-$ jita $[13,14]$, who derived formulas for the electrophoretic mobility of a porous sphere by introducing the Brinkman equation $[15,16]$ for the internal flow field of the particle and assuming that the double layer remains spherically symmetric in the presence of the applied electric field. The effect of the distortion of the counterion atmosphere around a nearly free-drained polyelectrolyte coil under an applied electric field was examined theoretically by Imai and Iwasa [17], and their numerical results agree well with the experimental data [18]. Recently, general expressions were derived for the electrophoretic mobility of a composite spherical particle which is a rigid colloidal sphere coated with a layer of porous substances or polymers at its surface [19-21]. This mobility expression tends to a formula obtained by Hermans and Fujita [13] for a spherical polyelectrolyte when the hard core of the composite particle vanishes and the electric potentials are low. However, the effects of particle charges on the diffusiophoretic velocity of porous particles have not yet been investigated.

In this paper, we analytically study the diffusiophoretic motion of a charged porous particle in an unbounded electrolyte solution. The density of charged segments of the porous particle is assumed to be uniform, but no assumption is made for the thickness of the double layer relative to the dimension of the particle. In the next section, we present the fundamental electrokinetic equations and boundary conditions which govern the electrolyte ion distributions, the electrostatic potential profile, and the fluid velocity field inside and outside the porous particle. These basic equations are linearized assuming that the ionic concentrations, the electric potential, and therefore the electrochemical potential energies of the ionic species have only slight deviations from equilibrium due to the motion of the particle. In Section 3, the axisymmetric diffusiophoretic motion of a charged porous sphere in a solution of a symmetrically charged electrolyte with a constant imposed concentration gradient is considered. The linearized electrokinetic equations are transformed into a set of differential equations by using a regular perturbation method with the density of the fixed charges inside the porous particle as the small perturbation parameter. The perturbed electrochemical potentials of ions, the perturbed electric potential, and the fluid flow field are determined by solving this set of differential equations subject to the appropriate boundary conditions. A closed-form expression for the diffusiophoretic velocity of the charged porous sphere is obtained from the balance between its electrostatic and hydrodynamic forces. Finally, typical numerical results of the diffusiophoretic mobility for the charged porous sphere are presented in Section 4.

\section{Basic electrokinetic equations for the diffusiophoresis of a charged porous particle}

We consider the diffusiophoretic motion of a charged porous sphere of radius $a$ immersed in an unbounded solution of a symmetrically charged binary electrolyte with a constant bulk concentration gradient $\nabla n^{\infty}$ in the $z$ direction, as illustrated in Fig. 1. The diffusiophoretic velocity of the particle is $U \mathbf{e}_{z}$, where $\mathbf{e}_{z}$ is the unit vector in the $z$ direction. The origin of the spherical coordinate system $(r, \theta, \phi)$ is taken at the center of the particle. Obviously, the problem is axially symmetric about the $z$-axis (or the polar axis $\theta=0)$.

It is assumed that the prescribed electrolyte concentration gradient is not high and hence that the system is only slightly distorted from equilibrium by the application of the gradient. Therefore, the ionic concentration distributions $n_{ \pm}(\mathbf{x})$ and the electric potential distribution $\psi(\mathbf{x})$ can be expressed as

$n_{ \pm}=n_{ \pm}^{(\mathrm{eq})}+\delta n_{ \pm}$

$\psi=\psi^{(\mathrm{eq})}+\delta \psi$

where $n_{ \pm}^{(\mathrm{eq})}(\mathbf{x})$ and $\psi^{(\mathrm{eq})}(\mathbf{x})$ are the equilibrium distributions of ionic concentrations and electrostatic potential, respectively, and $\delta n_{ \pm}(\mathbf{x})$ and $\delta \psi(\mathbf{x})$ are the small perturbations to the equilibrium state (in which no bulk concentration gradient or electric field is imposed). Here, the subscripts + and - refer to the cation and anion, respectively, and $\mathbf{x}$ is the position vector. The equilibrium concentration of each ionic species is related to the equilibrium potential by the Boltzmann distribution.

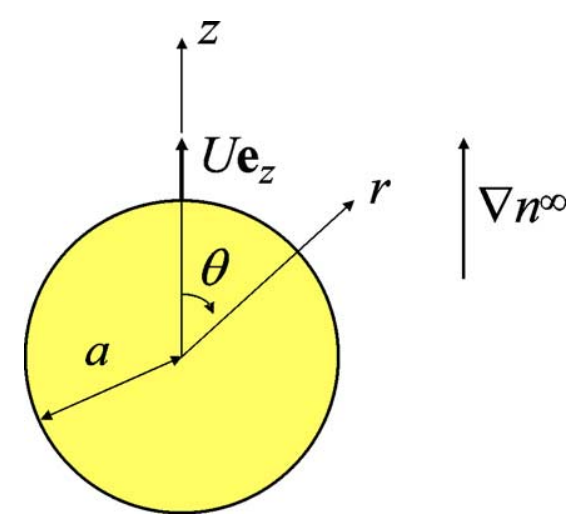

Fig. 1. Geometrical sketch for the diffusiophoresis of a charged porous sphere. 
It can be shown that the small perturbed quantities $\delta n_{ \pm}$ and $\delta \psi$ together with the fluid velocity field $\mathbf{u}(\mathbf{x})$ satisfy the following set of linearized electrokinetic equations $[11,21]$ :

$$
\begin{aligned}
& \nabla^{2} \nabla \times \mathbf{u}-h(\mathbf{x}) \lambda^{2} \nabla \times \mathbf{u} \\
& =-\frac{\varepsilon}{4 \pi \eta} \nabla \times\left(\nabla^{2} \psi^{(\mathrm{eq})} \nabla \delta \psi+\nabla^{2} \delta \psi \nabla \psi^{(\mathrm{eq})}\right), \\
& \nabla \cdot \mathbf{u}=0, \\
& \nabla^{2} \delta \mu_{ \pm}= \pm \frac{Z e}{k T}\left\{\nabla \psi^{(\mathrm{eq})} \cdot \nabla \delta \mu_{ \pm}\right. \\
& \left.-\frac{k T}{[1-(1-\omega) h(\mathbf{x})] D_{ \pm}} \nabla \psi^{(\mathrm{eq})} \cdot \mathbf{u}\right\}, \\
& \nabla^{2} \delta \psi=\frac{4 \pi Z e n^{\infty}(\mathbf{0})}{\varepsilon k T}\left[\exp \left(\frac{Z e \psi^{(\mathrm{eq})}}{k T}\right)\left(\delta \mu_{-}+Z e \delta \psi\right)\right. \\
& \left.-\exp \left(-\frac{Z e \psi^{(\mathrm{eq})}}{k T}\right)\left(\delta \mu_{+}-Z e \delta \psi\right)\right] \text {. }
\end{aligned}
$$

Here, $\delta \mu_{ \pm}(\mathbf{x})$ is defined as a linear combination of $\delta n_{ \pm}$and $\delta \psi$ based on the concept of the electrochemical potential energy [22],

$\delta \mu_{ \pm}=k T \frac{\delta n_{ \pm}}{n_{ \pm}^{(\mathrm{eq})}} \pm Z e \delta \psi$

$\eta$ is the viscosity of the fluid (the available evidence [23] suggests that it is reasonable to assume the same value of $\eta$ inside and outside the porous particle); $D_{ \pm}$and $\omega D_{ \pm}$(with $\omega \leqslant 1$ ) are the diffusion coefficients of the ionic species outside and inside the porous particle, respectively; $Z$ is the valence of the symmetric electrolyte, which is positive; $\lambda=(f / \eta)^{1 / 2}$, where $f$ is the hydrodynamic friction coefficient inside the porous particle per unit volume of the fluid (which accounts for the hindrance to the convective transport of the electrolyte caused by the frictional segments); $h(\mathbf{x})$ is a unit step function which equals unity if $\mathbf{x}$ is inside the particle, and zero otherwise; $\varepsilon=4 \pi \varepsilon_{0} \varepsilon_{\mathrm{r}}$, where $\varepsilon_{\mathrm{r}}$ is the relative permittivity of the electrolyte solution and $\varepsilon_{0}$ is the permittivity of a vacuum. We assume that the values of $\eta, f, \varepsilon, D_{ \pm}$, and $\omega$ are constant.

For counterions in polyelectrolyte networks, a theory for the ionic self-diffusion [24] and some experimental data [25] predict that the parameter $\omega$ (which is the normalized diffusion coefficient of the small ions in the porous structure) can have a value as low as $0.25-0.31$. Here, $\omega$ is taken to be the same for both ionic species. Note that $f$ can be expressed as $6 \pi \eta a_{S} N_{S}$ in the free-draining limit, where $N_{S}$ and $a_{S}$ are the number density and the Stokes radius, respectively, of the hydrodynamic frictional segments of the porous particle, and the reciprocal of the parameter $\lambda$ is the shielding length characterizing the extent of flow penetration inside the porous particle. For some model porous particles made of steel wool (in glycerin-water solution) [26] and plastic foam slab (in silicon oil) [27], experimental values of $1 / \lambda$ can be as high as $0.4 \mathrm{~mm}$, whereas in the surface regions of human erythrocytes [28], rat lymphocytes [29], and grafted polymer microcapsules [30] in salt solutions, values of $1 / \lambda$ were found to be about $3 \mathrm{~nm}$. Note that $1 / \lambda^{2}$ is the so-called "permeability" of the porous medium, which is related to its pore size and porosity and characterizes the dynamic behavior of the viscous fluid in it. Of course, the parameter $\omega$ may also depend on the pore size and porosity, especially when the pore size is small down to the order of the ionic size.

The conditions inside the porous particle are

$\mathbf{u}, \delta \mu_{ \pm}$, and $\delta \psi$ are finite.

The conditions far from the particle become

$$
\begin{aligned}
& r \rightarrow \infty: \quad \mathbf{u} \rightarrow-U \mathbf{e}_{z}, \\
& \delta \mu_{ \pm} \rightarrow k T(1 \mp \beta) \alpha \frac{r}{a} \cos \theta, \\
& \delta \psi \rightarrow-\frac{k T}{Z e} \beta \alpha \frac{r}{a} \cos \theta,
\end{aligned}
$$

where $\alpha=a\left|\nabla n^{\infty}\right| / n^{\infty}(\mathbf{0})$ and $\beta=\left(D_{+}-D_{-}\right) /\left(D_{+}+\right.$ $D_{-}$). Expression (8c) for the induced potential field, which arises spontaneously due to the imposed electrolyte gradient and the difference in mobilities of the cation and anion of the electrolyte, is derived from the requirement that the total fluxes of cations and anions are balanced in order to have no electric current generated in the electrically neutral bulk solution [1,7,31]. Equations (7a) and (8a) take a reference frame that the particle is at rest and the velocity of the fluid at infinity is the particle velocity in the opposite direction.

The boundary conditions at the particle surface $S$ are

$$
\begin{aligned}
& \left.\mathbf{u}\right|_{S^{+}}=\left.\mathbf{u}\right|_{S^{-}}, \\
& \left.\mathbf{n} \cdot \boldsymbol{\sigma}\right|_{S^{+}}=\left.\mathbf{n} \cdot \boldsymbol{\sigma}\right|_{S^{-}}, \\
& \left.\delta \mu_{ \pm}\right|_{S^{+}}=\left.\delta \mu_{ \pm}\right|_{S^{-}}, \\
& \left.\nabla \delta \mu_{ \pm}\right|_{S^{+}}=\left.\omega \nabla \delta \mu_{ \pm}\right|_{S^{-}}, \\
& \left.\delta \psi\right|_{S^{+}}=\left.\delta \psi\right|_{S^{-}}, \\
& \left.\nabla \delta \psi\right|_{S^{+}}=\left.\nabla \delta \psi\right|_{S^{-}} .
\end{aligned}
$$

Here, the superscripts + and - to $S$ represent the external and internal sides, respectively, to the surface of the particle, $\mathbf{n}$ is the unit vector outwardly normal to the particle surface, and $\sigma$ is the hydrodynamic stress of the fluid. Equations (9a) and (9b) are the continuity requirement of the fluid velocity and stress tensor at the particle surface which are physically realistic and mathematically consistent boundary conditions for the present problem [23,32,33]. Equations (9c) and (9d) state that the concentrations and fluxes of the ionic species must be continuous. Equations ( $9 \mathrm{e}$ ) and (9f) indicate that the potential and electric field are also continuous. The continuity of the electric field results from the assumption that the relative permittivity of the solution takes the same value both inside and outside the porous particle. In the present system, the total fluid stress, which consists of the hydrodynamic stress and the Maxwell stress, is continuous at the particle surface. The boundary condition for the continuity of hydrodynamic stress given by Eq. (9b) comes from the fact of continuous Maxwell stress that can be deduced from Eqs. (1b) and (9f) [20]. 


\section{Solution for the diffusiophoretic velocity}

The equilibrium electric potential $\psi^{(\mathrm{eq})}$ satisfies the Poisson-Boltzmann equation and appropriate boundary conditions (taking finite $\psi^{(\mathrm{eq})}$ inside the charged porous particle, continuous electric potential and electric field at the particle surface, and zero potential far from the particle). It is easy to show that, for an unbounded solution of a symmetric electrolyte surrounding a charged porous sphere with a uniform fixed charge density $Q$ inside the porous particle,

$\psi^{(\mathrm{eq})}(r)=\psi_{\mathrm{eq} 1} \bar{Q}+\mathrm{O}\left(\bar{Q}^{3}\right)$.

Here, $\bar{Q}=4 \pi Z e Q / \varepsilon \kappa^{2} k T$ is the nondimensional charge density of the porous particle, and

$\psi_{\mathrm{eq} 1}=\frac{k T}{Z e}\left[1-(\kappa a+1) e^{-\kappa a} \frac{\sinh (\kappa r)}{\kappa r}\right] \quad$ if $r \leqslant a$,
$\psi_{\mathrm{eq} 1}=\frac{k T}{Z e}[\kappa a \cosh (\kappa a)-\sinh (\kappa a)] \frac{e^{-\kappa r}}{\kappa r} \quad$ if $r \geqslant a$,

where the Debye screening parameter $\kappa=\left[8 \pi Z^{2} e^{2} n^{\infty}(\mathbf{0}) /\right.$ $\varepsilon k T]^{1 / 2}$. Expression (10) for $\psi^{(\mathrm{eq})}$ as a power series in $\bar{Q}$ up to $\mathrm{O}(\bar{Q})$ is the equilibrium solution for the linearized Poisson-Boltzmann equation that is valid for small values of the electric potential (the Debye-Hückel approximation). That is, the fixed charge density $Q$ of the particle must be small enough for the potential to remain small. Note that $\psi^{(\mathrm{eq})}$ and $n_{ \pm}^{(\mathrm{eq})}$ depend on $r$ only due to spherical symmetry, and the contribution from the effects of $\mathrm{O}\left(\bar{Q}^{2}\right)$ to $\psi^{(\mathrm{eq})}$ disappears only for the case of symmetric electrolytes.

To solve the small quantities $\mathbf{u}, \delta \mu_{ \pm}$, and $\delta \psi$ in terms of the particle velocity $U$ when the parameter $\bar{Q}$ is small, these variables can be written as regular perturbation expansions in powers of $\bar{Q}$,

$$
\begin{aligned}
& \mathbf{u}=\mathbf{u}_{1} \bar{Q}+\mathbf{u}_{2} \bar{Q}^{2}+\cdots, \\
& \delta \mu_{ \pm}=\mu_{0 \pm}+\mu_{1 \pm} \bar{Q}+\mu_{2 \pm} \bar{Q}^{2}+\cdots, \\
& \delta \psi=\psi_{0}+\psi_{1} \bar{Q}+\psi_{2} \bar{Q}^{2}+\cdots, \\
& U=U_{1} \bar{Q}+U_{2} \bar{Q}^{2}+\cdots,
\end{aligned}
$$

where the functions $\mathbf{u}_{i}, \mu_{i \pm}, \psi_{i}$, and $U_{i}$ are independent of $\bar{Q}$. The zeroth-order terms of $\mathbf{u}$ and $U$ disappear because an uncharged particle will not move by imposing an electrolyte concentration gradient if only the electrostatic interaction is considered.

Substituting the expansions given by Eq. (12) and $\psi^{(\mathrm{eq})}$ given by Eq. (10) into the governing Eqs. (2)-(5) and boundary conditions (7)-(9), and equating like powers of $\bar{Q}$ on both sides of the respective equations, we can obtain a set of linear differential equations and boundary conditions for each set of the functions $\mathbf{u}_{i}, \mu_{i \pm}$, and $\psi_{i}$ with $i$ equal to 0,1 , and 2. After solving these perturbation equations, the results for the $r$ and $\theta$ components of $\mathbf{u}$ (to the order of $\bar{Q}^{2}$ ), $\delta \mu_{ \pm}$, and $\delta \psi$ (to the order of $\bar{Q}$ ) can be written as

$$
\begin{aligned}
u_{r}=\{ & \left\{U_{1} F_{0}(r)-\frac{k T}{\eta a^{2}} \beta \alpha F_{1}(r)\right] \bar{Q} \\
& \left.+\left[U_{2} F_{0}(r)+\frac{k T}{\eta a^{2}} \alpha F_{2}(r)\right] \bar{Q}^{2}\right\} \cos \theta, \\
u_{\theta}= & -\frac{1}{2 r} \frac{d}{d r}\left\{r^{2}\left[U_{1} F_{0}(r)-\frac{k T}{\eta a^{2}} \beta \alpha F_{1}(r)\right] \bar{Q}\right. \\
& \left.+r^{2}\left[U_{2} F_{0}(r)+\frac{k T}{\eta a^{2}} \alpha F_{2}(r)\right] \bar{Q}^{2}\right\} \sin \theta, \\
\delta \mu_{ \pm}= & k T(1 \mp \beta) \alpha\left[F_{\mu 0}(r) \mp F_{\mu 1}(r) \bar{Q}\right] \cos \theta, \\
\delta \psi= & \frac{k T}{Z e} \alpha\left[-\beta F_{\psi 0}(r)+F_{\psi 1}(r) \bar{Q}\right] \cos \theta .
\end{aligned}
$$

Here, $F_{i}(r)$ (with $i$ equal to 0,1 , and 2), $F_{\mu 0}(r), F_{\psi 0}(r)$, $F_{\mu 1}(r)$, and $F_{\psi 1}(r)$ are dimensionless functions of $r$ defined in Appendix A. Note that the solutions for $\delta \mu_{ \pm}$and $\delta \psi$ to the order of $\bar{Q}$, which will be sufficient for the calculation of the particle velocity to $\mathrm{O}\left(\bar{Q}^{2}\right)$, do not contain the influence of the fluid motion.

The total force exerted on a charged porous sphere undergoing diffusiophoresis in an electrolyte solution can be expressed as the sum of the electrostatic force and the hydrodynamic friction force. The electric force acting on the porous sphere is defined by

$\mathbf{F}_{\mathrm{e}}=-\int_{r \leqslant a} Q \nabla \psi d \mathbf{x}$.

Substituting Eqs. (1b) and (15) into Eq. (16), and using the fact that the net electric force acting on the particle at the equilibrium state is zero, one has

$$
\begin{aligned}
\mathbf{F}_{\mathrm{e}}=\frac{\varepsilon}{3}\left(\frac{\kappa a k T}{Z e}\right)^{2} \alpha[ & \beta F_{\psi 0}(a) \bar{Q} \\
& \left.-F_{\psi 1}(a) \bar{Q}^{2}+\mathrm{O}\left(\bar{Q}^{3}\right)\right] \mathbf{e}_{z} .
\end{aligned}
$$

The hydrodynamic friction force acting on the porous sphere is given by

$$
\mathbf{F}_{\mathrm{h}}=\int_{r \leqslant a} f \mathbf{u}(\mathbf{x}) d \mathbf{x} .
$$

Substitution of Eq. (13) into the above equation results in

$$
\begin{aligned}
\mathbf{F}_{\mathrm{h}}=\frac{4 \pi}{3} \eta \lambda^{2} a^{3}\{ & {\left[U_{1} F_{0}(a)-\frac{k T}{\eta a^{2}} \beta \alpha F_{1}(a)\right] \bar{Q} } \\
& \left.+\left[U_{2} F_{0}(a)+\frac{k T}{\eta a^{2}} \alpha F_{2}(a)\right] \bar{Q}^{2}\right\} \mathbf{e}_{z} .
\end{aligned}
$$

At steady state, the total force acting on the diffusiophoretic particle is zero. Applying this constraint to the summation of Eqs. (17) and (19) for a symmetric electrolyte, one 
obtains

$U_{1}=\frac{\varepsilon \beta \alpha}{4 \pi \eta a}\left(\frac{k T}{Z e}\right)^{2}(\kappa a)^{2} H_{1}$,

$U_{2}=\frac{\varepsilon \alpha}{4 \pi \eta a}\left(\frac{k T}{Z e}\right)^{2}(\kappa a)^{4} H_{2}$,

where $H_{1}$ and $H_{2}$ are dimensionless functions of $\kappa a$ and $\lambda a$ defined by

$H_{1}=\frac{4 \pi(Z e)^{2}}{\varepsilon a k T} \frac{1}{(\kappa a)^{2}} \frac{F_{1}(a)}{F_{0}(a)}-\frac{1}{(\lambda a)^{2}} \frac{F_{\psi 0}(a)}{F_{0}(a)}$,

$H_{2}=-\frac{4 \pi(Z e)^{2}}{\varepsilon a k T} \frac{1}{(\kappa a)^{4}} \frac{F_{2}(a)}{F_{0}(a)}+\frac{1}{(\kappa a \lambda a)^{2}} \frac{F_{\psi 1}(a)}{F_{0}(a)}$.

From Eqs. (12d) and (20), the diffusiophoretic velocity of the charged porous sphere can be expressed as

$$
\begin{aligned}
U=\frac{\varepsilon \alpha}{4 \pi \eta a}\left(\frac{k T}{Z e}\right)^{2}[ & \beta(\kappa a)^{2} H_{1} \bar{Q} \\
& \left.+(\kappa a)^{4} H_{2} \bar{Q}^{2}+\mathrm{O}\left(\bar{Q}^{3}\right)\right] .
\end{aligned}
$$

Note that $(\kappa a)^{2} \bar{Q}\left(=4 \pi a^{2} \mathrm{ZeQ} / \varepsilon k T\right)$ is independent of $\kappa$ or $n^{\infty}(\mathbf{0})$ for a constant fixed charge density $Q$. Since the solutions for $\delta \mu_{ \pm}$and $\delta \psi$ given by Eqs. (14) and (15) are not influenced by the fluid flow, the effect of the polarization (or relaxation) of the diffuse ions in the electric double layer surrounding the particle is not included in Eq. (20) up to the order $\bar{Q}^{2}$.

For the limiting case of a charged porous sphere with $\lambda a \rightarrow \infty$ (very high density of the hydrodynamic frictional segments) and $\omega=1$ (equal ionic diffusivities inside and outside the particle), Eq. (21) reduces to

$$
\begin{aligned}
H_{1}= & \frac{2}{3}(\kappa a)^{-3} e^{-\kappa a} \gamma(\kappa a), \\
H_{2}= & \frac{1}{9}(\kappa a)^{-3} \gamma(\kappa a)\left[E_{5}(\kappa a)-E_{3}(\kappa a)\right] \\
& +\frac{1}{12}(\kappa a)^{-6} e^{-2 \kappa a}[\gamma(\kappa a)]^{2},
\end{aligned}
$$

where

$E_{n}(x)=\int_{1}^{\infty} t^{-n} e^{-x t} d t$

and $\gamma(x)$ is defined by Eq. (A.5a) in Appendix A. On the other hand, when $\kappa a=0$ and $\omega=1$, Eq. (21) reduces to

$H_{1}=\left[9 A \gamma(\lambda a)(\lambda a)^{2}\right]^{-1}$

and $H_{2}=0$.

\section{Results and discussion}

Because all the governing equations and boundary conditions in this analysis have been linearized, diffusiophoresis of a charged porous particle in an electrolyte solution can be considered as a linear combination of two effects: (i) chemiphoresis due to the nonuniform adsorption of counterions and depletion of co-ions over the external and internal surfaces of the particle, which is analogous to diffusiophoresis in nonionic media [3,10], and (ii) electrophoresis due to the macroscopic electric field generated by the electrolyte concentration gradient given by Eq. (8c). In Eq. (22) for the diffusiophoretic velocity, the $\mathrm{O}(\bar{Q})$ term (involving parameter $\beta$ ) results from the contribution of electrophoresis, while the $\mathrm{O}\left(\bar{Q}^{2}\right)$ term represents the chemiphoretic contribution. Note that, when $\omega=1$, the function $H_{1}$ given by Eq. (21a) is the same as that obtained by Hermans and Fujita [13] for the electrophoretic mobility of a porous sphere.

Figs. 2-4 show plots of the first-order coefficient $H_{1}$ in Eq. (22) for the diffusiophoretic velocity of a charged porous sphere calculated using Eq. (21a) as a function of the para-

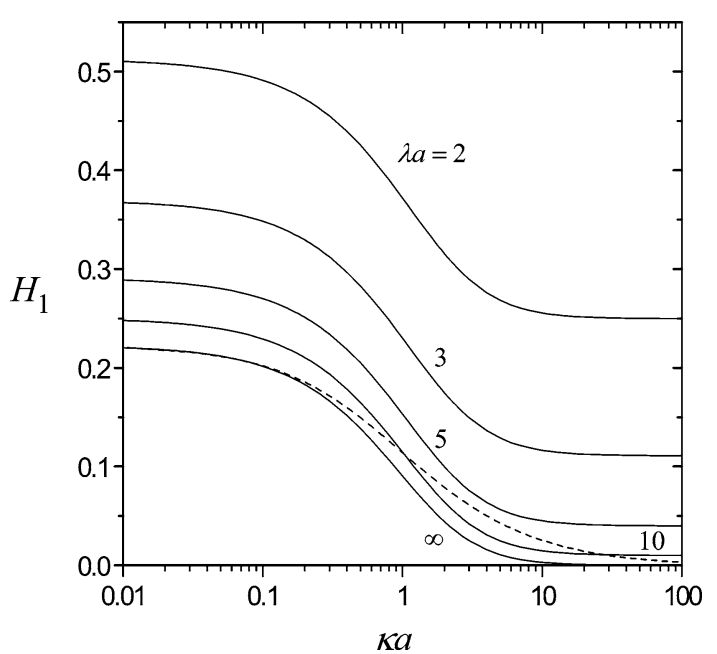

(a)

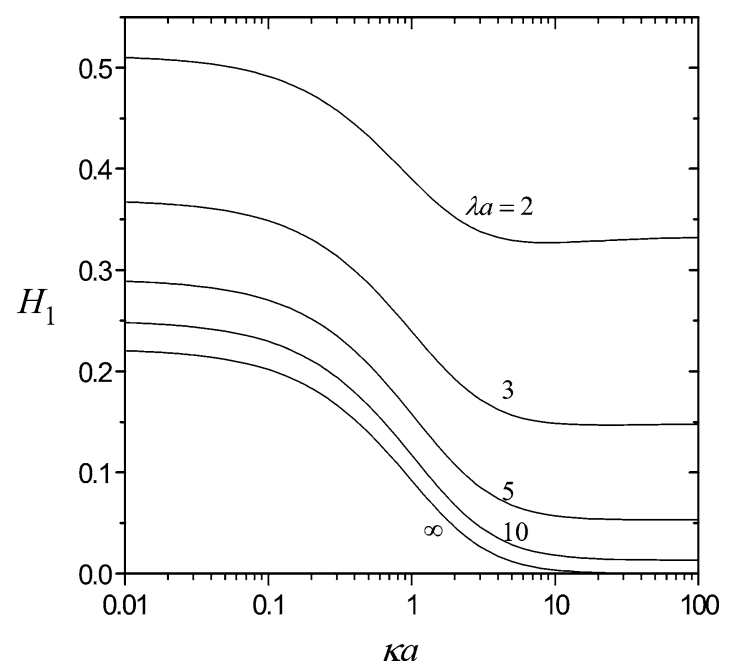

(b)

Fig. 2. Plots of the coefficient $H_{1}$ given by Eq. (21a) for the diffusiophoretic/electrophoretic velocity of a charged porous sphere versus $\kappa a$ at fixed values of $\lambda a$ : (a) $\omega=1$; (b) $\omega=0.25$. The dashed curve is plotted for an impermeable sphere having the same total charge (on its surface) as a porous sphere of equal radius. 


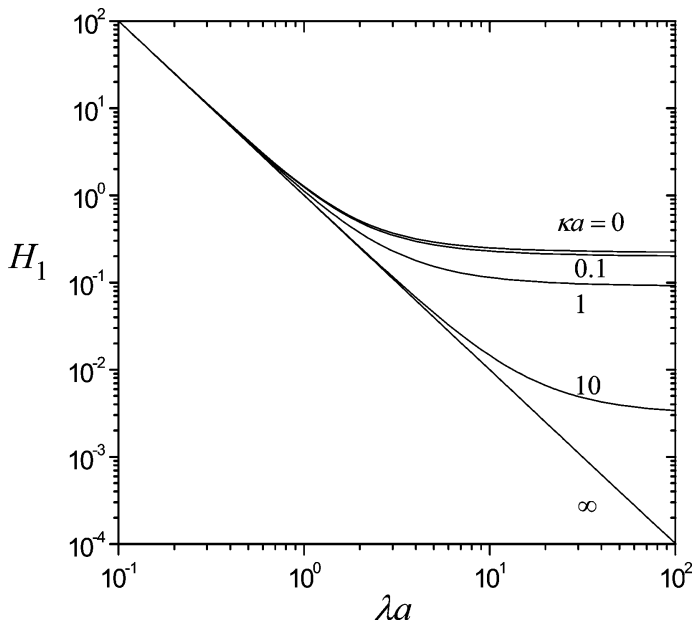

(a)

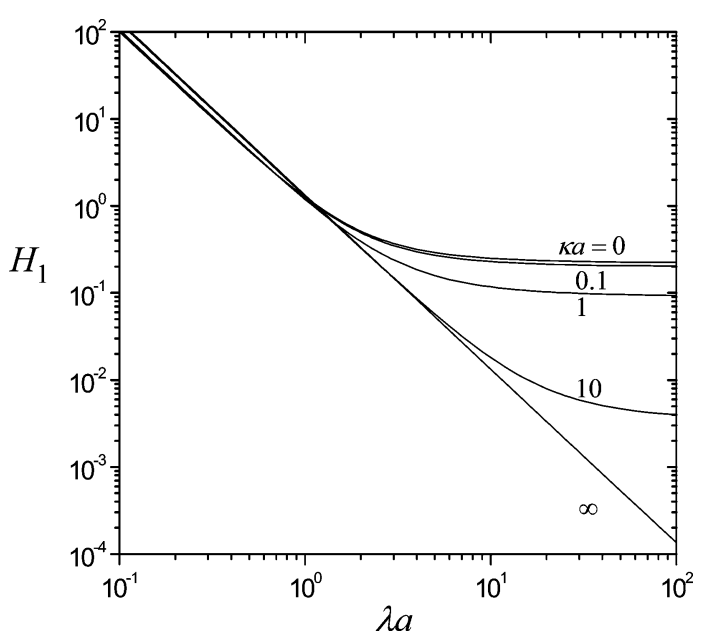

(b)

Fig. 3. Plots of the coefficient $H_{1}$ given by Eq. (21a) for the diffusiophoretic/electrophoretic velocity of a charged porous sphere versus $\lambda a$ at fixed values of $\kappa a$ : (a) $\omega=1$; (b) $\omega=0.25$.

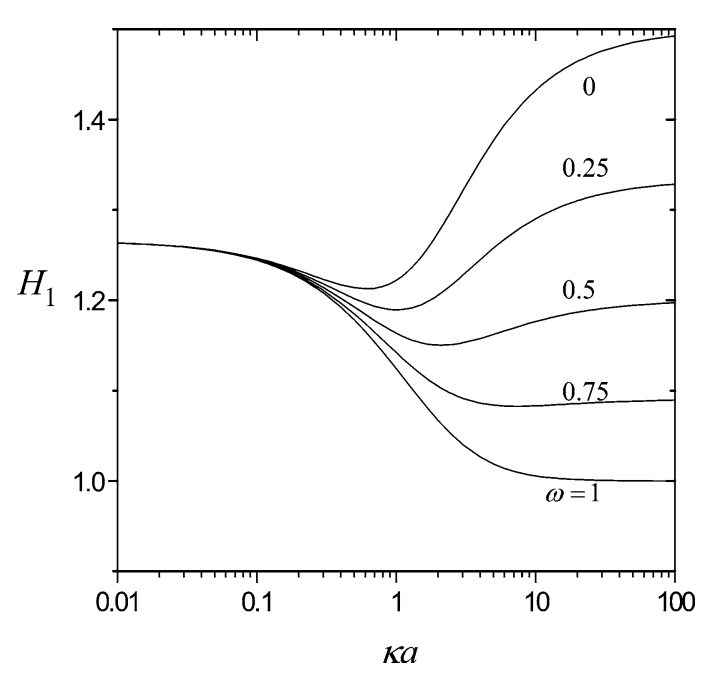

Fig. 4. Plots of the coefficient $H_{1}$ given by Eq. (21a) for the diffusiophoretic/electrophoretic velocity of a charged porous sphere versus $\kappa a$ for various values of $\omega$ at $\lambda a=1$. meters $\kappa a, \lambda a$, and $\omega$. As expected, $H_{1}$ is always a positive value and thus the direction of the particle motion caused by electrophoresis is determined by the sign of the product of the parameter $\beta$ (which determines the direction of the induced electric field according to Eq. (8c)) and the fixed charge density $Q$. Also, $H_{1}$ increases monotonically with decreasing $\lambda a$ for given values of $\kappa a$ and $\omega$.

For the diffusiophoretic velocity of an impermeable (nonporous) dielectric sphere with a uniform charge distribution at its (external) surface, an expansion solution in the form of Eq. (22) (in which $\bar{Q}$ can be taken as the dimensionless surface charge density $(\bar{\sigma}=4 \pi Z e \sigma / \varepsilon \kappa k T)$ or zeta potential $(\bar{\zeta}=Z e \zeta(\kappa a+1) / \kappa a k T)$ of the impermeable sphere having the same total fixed charge as a porous sphere of equal radius (i.e., taking $\sigma=Q a / 3$ )) was developed [11]. The coefficient $H_{1}$ for the impermeable charged sphere as a function of $\kappa a$ is drawn by a dashed curve in Fig. 2a for comparison. It can be seen that the values of $H_{1}$ for the impermeable sphere are comparable with those for the porous sphere (taking $\omega=1$ ) with $\lambda a \rightarrow \infty$ when $\kappa a<0.3$ or $>100$ but are equivalent to those for the porous sphere with $\lambda a=10$ when $\kappa a \approx 0.1$ or 25 . Note that, although the coefficient $H_{1}$ for the impermeable sphere decreases with increasing $\kappa a$, its conventional dimensionless electrophoretic mobility in an external electric field, which equals $3(\kappa a+1) H_{1}$, is a monotonic increasing function of $\kappa a[11,34]$.

When $\omega=1$, as shown in Figs. 2a and 3a, the value of $H_{1}$ for the diffusiophoresis/electrophoresis of a charged porous sphere decreases monotonically with an increase in $\kappa a$ (as for the case of an impermeable dielectric sphere) for a specified value of $\lambda a$, the dependence of $H_{1}$ on $\kappa a$ becomes weak if $\lambda \alpha<1$, and $H_{1} \rightarrow(\lambda a)^{-2}$ as $\lambda a \rightarrow 0$ or $\kappa a \rightarrow \infty$ (which can be derived from Eq. (21a)). When $\omega<1$, such as for the case of $\omega=0.25$ illustrated in Figs. $2 \mathrm{~b}$ and $3 \mathrm{~b}, H_{1}$ is still a monotonic decreasing function of $\kappa a$ for a large given value of $\lambda a$ (say, $\lambda a>3$ ), but becomes a monotonic increasing function of $\kappa a$ for a small fixed value of $\lambda a$ (say, $\lambda a<0.5$ ). For an intermediate value of $\lambda a$, such as the case of $\lambda a=1$ given in Fig. 4, $H_{1}$ decreases first, reaches a minimum, and then increases monotonically as $\kappa a$ increases from zero to infinity. The location of the minimum shifts to smaller $\kappa a$ as $\omega$ decreases. It can be seen that, for given values of $\kappa a$ and $\lambda a$, the coefficient $H_{1}$ increases with a decrease in the value of $\omega$ (i.e., the electrophoretic mobility of a charged porous sphere becomes greater as the hindrance to the diffusive transport of the electrolyte inside the particle is more significant). The dependence of $H_{1}$ on $\omega$ can be strong as $\kappa a>1$, but becomes quite weak as $\kappa a<0.1$ (or as $\lambda a$ is large).

The fact that the coefficient $H_{1}$ is hardly dependent on $\omega$ at small $\kappa a$ can be physically explained. At small $\kappa a$, the porous particle behaves electrically like a point charge, so the detail of what happens (such as the diffusion of ions) inside the particle is unimportant for the determination of its mobility by a balance between the electrostatic and the hydrodynamic forces. This argument applies to the secondorder coefficient $\mathrm{H}_{2}$ in Eq. (22) as well. 


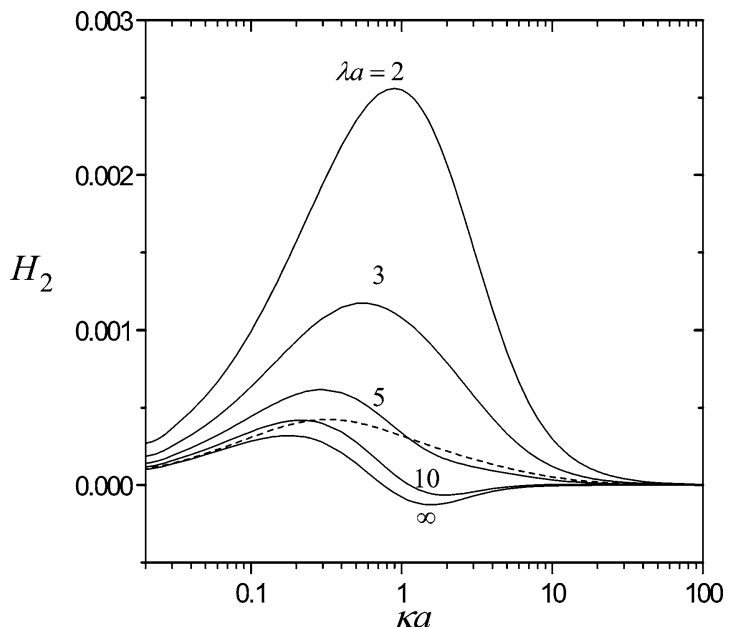

(a)

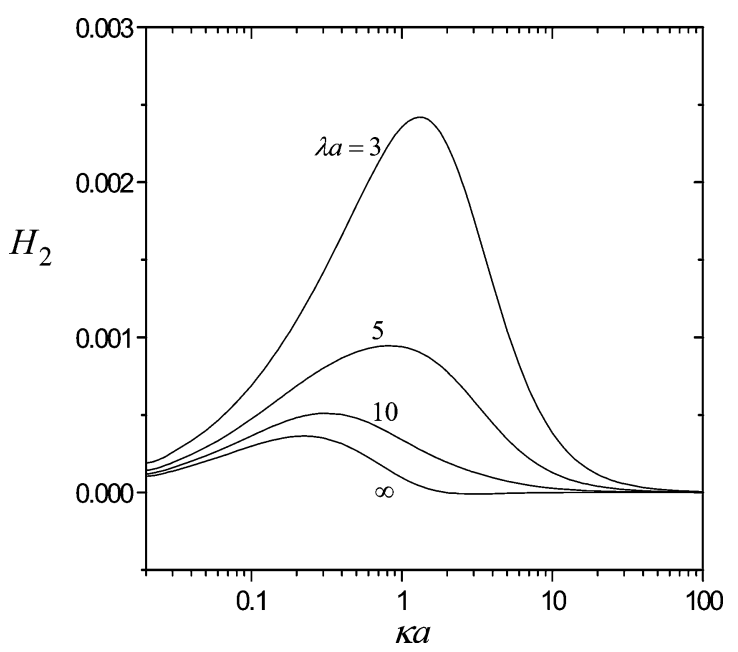

(b)

Fig. 5. Plots of the coefficient $H_{2}$ given by Eq. (21b) for the diffusiophoretic velocity of a charged porous sphere versus $\kappa a$ at fixed values of $\lambda a$ : (a) $\omega=1$; (b) $\omega=0.25$. The dashed curve is plotted for an impermeable sphere having the same total charged (on its surface) as a porous sphere of equal radius.

The coefficient $H_{2}$ for the diffusiophoretic velocity of a charged porous sphere can be calculated using Eq. (21b) and its results as a function of the parameters $\kappa a, \lambda a$, and $\omega$ are plotted in Figs. 5-7. The corresponding results for an impermeable dielectric sphere [11] having the same total charge (on its surface) as a porous sphere of equal radius (taking $\bar{Q}=3 \bar{\sigma} / \kappa a$ in Eq. (22)) as a function of $\kappa a$ are also displayed in Fig. 5a for comparison. Analogous to (but slightly different from) the case with $H_{1}$, the values of $\mathrm{H}_{2}$ for the impermeable sphere are comparable with those for the porous sphere (taking $\omega=1$ ) with $\lambda a \rightarrow \infty$ when $\kappa a<0.1$ and are close to those for the porous sphere with $\lambda a=5$ when $\kappa a>1$. Although the coefficient $H_{2}$ for the impermeable sphere is not a monotonic function of $\kappa a$, its conventional dimensionless chemiphoretic mobility, which equals $72(\kappa a+1)^{2} H_{2}$, increases monotonically with an increase in $\kappa a$ [11]. Again, $H_{2}$ increases monotonically with

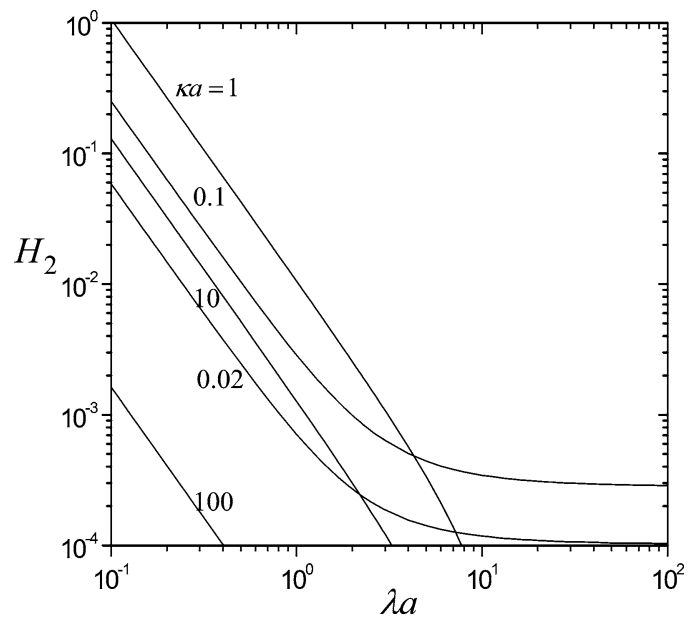

(a)

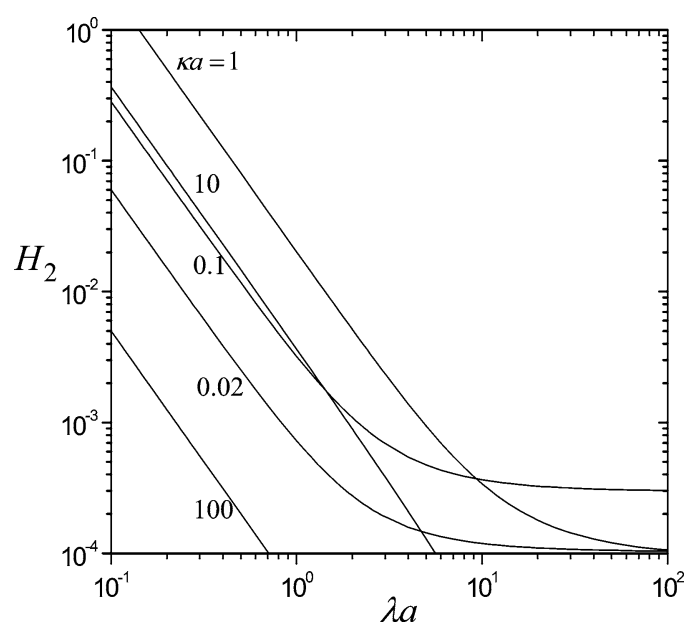

(b)

Fig. 6. Plots of the coefficient $H_{2}$ given by Eq. (21b) for the diffusiophoretic velocity of a charged porous sphere versus $\lambda a$ at fixed values of $\kappa a$ : (a) $\omega=1$; (b) $\omega=0.25$.

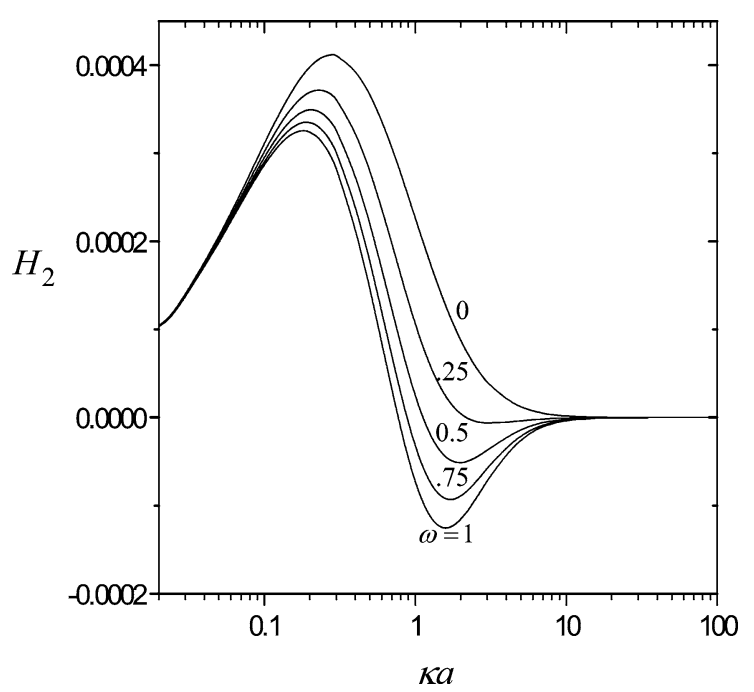

Fig. 7. Plots of the coefficient $H_{2}$ given by Eq. (21b) for the diffusiophoretic velocity of a charged porous sphere versus $\kappa a$ for various values of $\omega$ at $\lambda a=100$. 
a decrease in $\lambda a$ for fixed values of $\kappa a$ and $\omega$. Note that the value of $\mathrm{H}_{2}$ is about two orders of magnitude smaller than that of $H_{1}$.

For specified values of $\lambda a$ and $\omega$, as shown in Fig. 5, the value of $\mathrm{H}_{2}$ (the normalized chemiphoretic mobility of the porous sphere) is maximal at some value of $\kappa a$ between 0.1 and 2, and fades out as the value of $\kappa a$ gets small or large. Both the limits $\kappa a \rightarrow 0$ and $\kappa a \rightarrow \infty$ result in $H_{2}=0$ (but the contribution from the chemiphoretic effect vanishes only for the case of $\kappa a \rightarrow 0$ ). Interestingly, when the value of $\lambda a$ is sufficiently large (e.g., $\lambda a>10$ for the case of $\omega=1$ ), $H_{2}$ decreases first, reaches a minimum which is negative, and then increases monotonically to zero as $\kappa a$ increases from the value at the maximal point of $H_{2}$ to infinity. The locations of the minimum (if it exists) and maximum in $\mathrm{H}_{2}$ shift to smaller $\kappa a$ as $\lambda a$ increases. Fig. 6 indicates that, for given values of $\kappa a$ and $\omega$, the value of $H_{2}$ approaches infinity in proportion to $(\lambda a)^{-2}$ as $\lambda a \rightarrow 0$. For fixed values of $\kappa a$ and $\lambda a$, as illustrated in Fig. 7 , the coefficient $H_{2}$ increases with decreasing value of $\omega$, similar to the trend of the coefficient $H_{1}$, and the locations of the maximum and minimum in $H_{2}$ shift to greater $\kappa a$ as $\omega$ decreases.

For the diffusiophoresis/electrophoresis of a porous particle, the diffusion of the solute species in the fluid solution affects the particle's movement through two mechanisms. Obviously, the concentration gradient of the solute along the external surface of the particle leads to a diffusioosmotic/electroosmotic flow, which drives the porous particle to move in the same direction as that for the corresponding motion of an impermeable particle under an otherwise identical condition. On the other hand, the diffusion of the solute species inside the porous particle drags the surrounding fluid which then drives the porous particle to move in a direction opposite to that for the diffusiophoresis/electrophoresis of the impermeable particle. The combination of the two mechanisms results in the fact that both the coefficients $H_{1}$ and $\mathrm{H}_{2}$ increase with a decrease in the parameter $\omega$ (the relative diffusion coefficient of the solute species in the porous structure). Evidently, for some cases of the part of chemiphoresis (as the value of $\kappa a$ has the order of unity), the contribution from the diffusion of the solute inside the porous particle can be dominant over the contribution from the osmotic flow along the external surface of the particle so that the value of $\mathrm{H}_{2}$ is negative. On the other hand, the coefficient $\mathrm{H}_{2}$ becomes hardly dependent on the parameter $\omega$ when the value of $\kappa a$ gets large or small, as it is shown in Fig. 7.

In Fig. 8, the dependence of the diffusiophoretic velocity of a charged porous sphere on its dimensionless fixed charge density $(\kappa a)^{2} \bar{Q}=4 \pi a^{2} Z e Q / \varepsilon k T$ at various values of $\kappa a, \lambda a$, and $\omega$ for the case that the cation and anion diffusivities are equal $(\beta=0)$ is shown. The magnitude of the diffusiophoretic velocity is normalized by a characteristic value given by

$U^{*}=\frac{\varepsilon \alpha}{4 \pi \eta a}\left(\frac{k T}{Z e}\right)^{2}$.

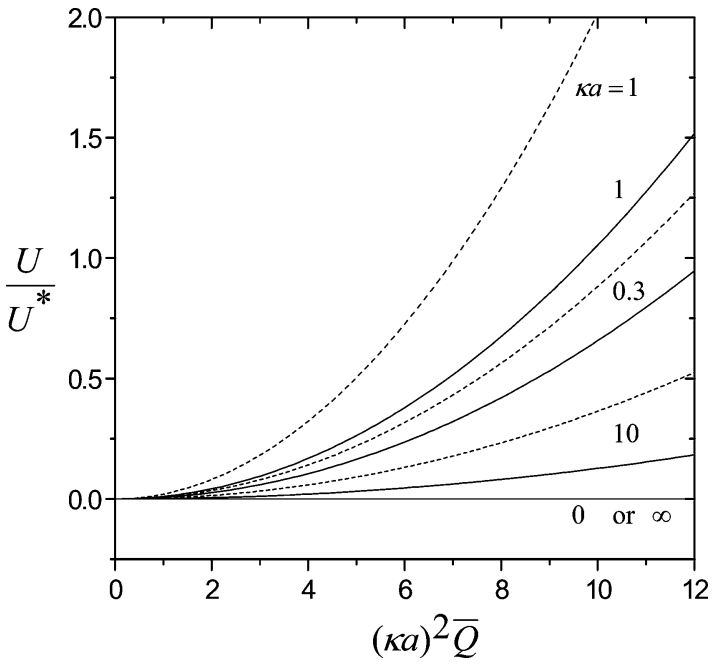

(a)

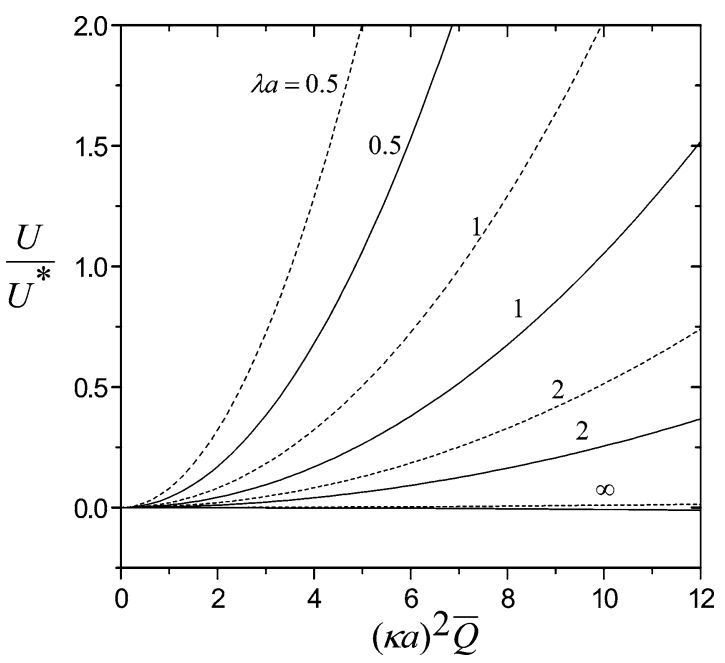

(b)

Fig. 8. Plots of the normalized diffusiophoretic mobility of a charged porous sphere in a symmetric electrolyte solution with $\beta=0$ versus the dimensionless fixed charge density: (a) $\lambda a=1$; (b) $\kappa a=1$. The solid and dashed curves denote the cases of $\omega=1$ and $\omega=0.25$, respectively.

Only the results at positive $\bar{Q}$ are displayed in Fig. 8 since the particle velocity, which is due to the chemiphoretic effect entirely for the case $\beta=0$, is an even function of $\bar{Q}$ as illustrated by Eq. (22). Because our analysis is based on the assumption of small electric potential or fixed charged density, the magnitudes of $(\kappa a)^{2} \bar{Q}$ considered are less than 12 , which is equivalent to the case of an impermeable dielectric sphere with $\kappa a$ in the order of unity having the magnitudes of its normalized zeta potential $(Z e \zeta / k T$ ) less than 2. Evidently, in this range of $(\kappa a)^{2} \bar{Q}$, the reduced diffusiophoretic velocity $U / U^{*}$ increases monotonically with an increase in $(\kappa a)^{2} \bar{Q}$ for given values of $\kappa a, \lambda a$, and $\omega$, with a decrease in $\lambda a$ for specified values of $\kappa a, \omega$, and $\bar{Q}$, and with a decrease in $\omega$ for constant values of $\kappa a, \lambda a$, and $\bar{Q}$. For fixed values of $\lambda a, \omega$, and $(\kappa a)^{2} \bar{Q}$, the value of $U / U^{*}$ is maximal at a finite value of $\kappa a$, and decreases as $\kappa a$ increases or decreases from this finite value. There is no chemiphoretic motion of the par- 


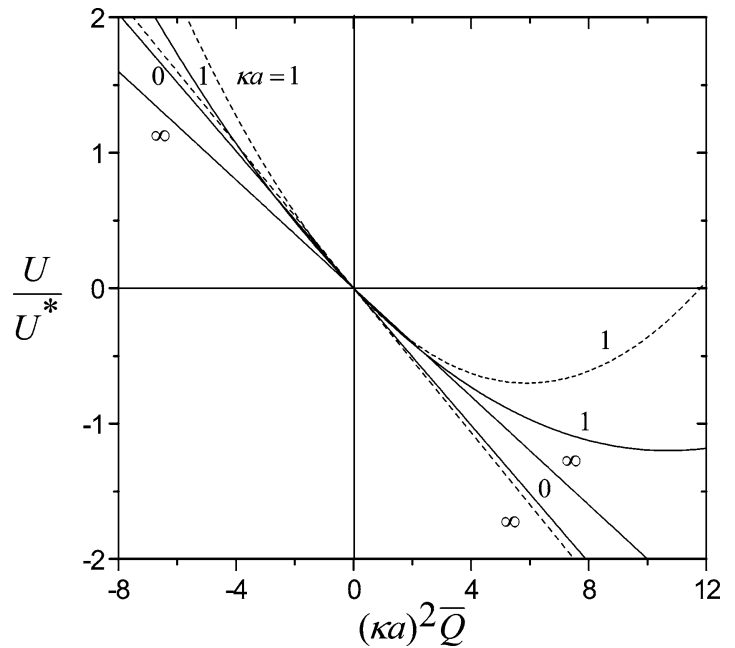

(a)

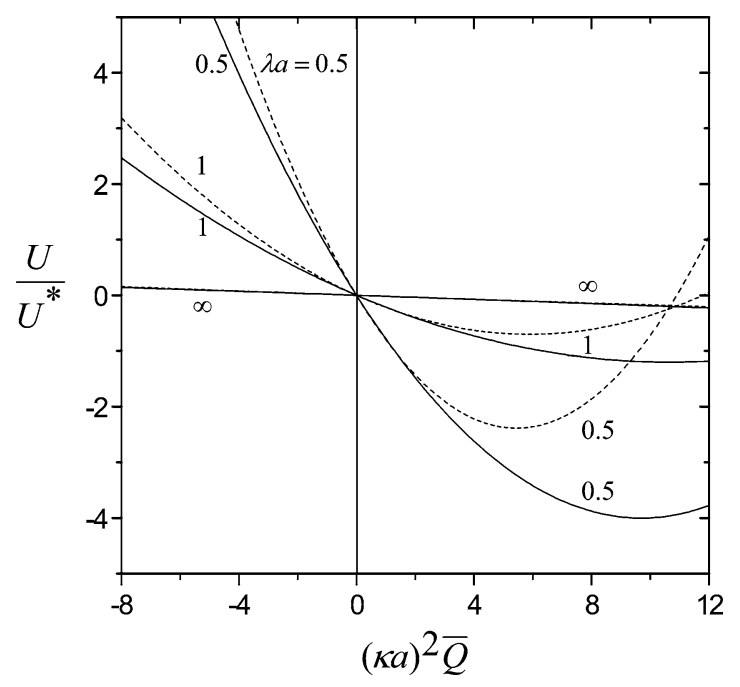

(b)

Fig. 9. Plots of the normalized diffusiophoretic mobility of a charged porous sphere in a symmetric electrolyte solution with $\beta=-0.2$ versus the dimensionless fixed charge density: (a) $\lambda a=1$; (b) $\kappa a=1$. The solid and dashed curves denote the cases of $\omega=1$ and $\omega=0.25$, respectively.

ticle for the limiting cases of $\bar{Q}=0, \kappa a=0$, or $\kappa a \rightarrow \infty$. If the magnitude of $\lambda a$ is sufficiently large, the value of $U / U^{*}$ may become negative (the particle reverses its direction of movement) at some values of $\kappa a$.

Fig. 9 is drawn for the normalized diffusiophoretic velocity of the porous sphere as a function of $(\kappa a)^{2} \bar{Q}$ at various values of $\kappa a, \lambda a$, and $\omega$ for a case where the cation and anion have different diffusion coefficients $(\beta=-0.2)$. In this case, both the electrophoretic and the chemiphoretic effects contribute to the motion of the particle and the net diffusiophoretic velocity is neither an even nor an odd function of $\bar{Q}$. Again, for given values of $\lambda a, \omega$, and $(\kappa a)^{2} \bar{Q}$, the normalized diffusiophoretic velocity $U / U^{*}$ is not a monotonic function of $\kappa a$. In the limits of $\kappa a=0$ and $\kappa a \rightarrow \infty$, the chemiphoretic contribution disappears and Eq. (22) for the particle velocity leads to straight lines in Fig. 9a due to the electrophoretic contribution only. For some intermediate val- ues of $\kappa a$, the normalized particle velocity $U / U^{*}$ is not necessarily a monotonic function of $(\kappa a)^{2} \bar{Q}$. Some of the curves in Fig. 9 show that the particle might reverse direction of movement more than once as its dimensionless fixed charge density varies from negative to positive values. The reversals occurring at the values of $(\kappa a)^{2} \bar{Q}$ other than zero result from the competition between the contributions from electrophoresis and chemiphoresis. Note that the situations associated with Figs. 8 and 9 (taking $Z=1$ ) are close to the diffusiophoresis in the aqueous solutions of $\mathrm{KCl}$ and $\mathrm{NaCl}$, respectively.

\section{Concluding remarks}

In this paper, the steady-state diffusiophoresis of a charged porous sphere with arbitrary values of the parameters $\kappa a, \lambda a$, and $\omega$ in an unbounded solution of a symmetric electrolyte with a uniform imposed concentration gradient is analyzed. The porous particle is treated as a solventpermeable and ion-penetrable object in which fixed-charged groups and frictional segments are distributed at uniform densities. Solving the linearized continuity equations of ions, Poisson-Boltzmann equation, and modified StokesBrinkman equations applicable to the system by a regular perturbation method, we have obtained the ion concentration (or electrochemical potential energy) distributions, the electric potential profile, and the fluid velocity field. The requirement that the total force exerted on the particle be zero leads to Eqs. (21) and (22) for the diffusiophoretic velocity of the charged porous sphere as a function of the parameters $\kappa a, \lambda a$, and $\omega$ correct to the order $\bar{Q}^{2}$.

Equations (21) and (22) are obtained on the basis of the Debye-Hückel approximation for the equilibrium potential distribution around the porous sphere. A similar formula for the electrophoretic velocity of an impermeable sphere with a low zeta potential was shown to give an excellent approximation for the case of reasonably high zeta potential (with errors less than $4 \%$ for $|\zeta| e / k T \leqslant 2$ ) [35]. Also, comparing with the numerical solution for the diffusiophoretic mobility of an impermeable sphere in $\mathrm{KCl}$ and $\mathrm{NaCl}$ aqueous solutions obtained by Prieve and Roman [8] valid for an arbitrary value of zeta potential, we found that the expansion formula for the sphere with low zeta potential is also quite accurate for the entire range of $|\zeta| e / k T \leqslant 2$ [11]. Therefore, our results in Eqs. (21) and (22) might be used tentatively for the situation of high electric potentials. On the other hand, the decay of the density distributions of frictional segments and fixed charges in the porous particle with the distance from the particle center has not been considered in our calculations. In order to see whether our theory can be reasonably extended to the higher values of electric potential or to the nonuniform density distributions of frictional segments and fixed charges, we propose to obtain a numerical solution of the electrokinetic differential equations with no assumption on the magnitude of electric potential allowing the use of 
arbitrary distributions of fixed charge and fluid drag components in the porous particle and compare it with the approximate solution.

\section{Acknowledgment}

This research was supported by the National Science Council of the Republic of China under Grant NSC91-2214E-002-012.

\section{Appendix A}

For conciseness the definitions of some functions in Section 3 are listed here. In Eq. (13),

$$
\begin{aligned}
F_{0}(r)= & -3 A \gamma(\lambda a)-6 A \gamma(\lambda r)\left(\frac{a}{r}\right)^{3} \text { if } r<a \\
F_{0}(r)= & -1+3(\lambda a)^{2} A \gamma(\lambda a) \frac{a}{r}-A B\left(\frac{a}{r}\right)^{3} \text { if } r>a \\
F_{i}(r)= & C_{i 1}+C_{i 2} \gamma(\lambda r)\left(\frac{a}{r}\right)^{3} \\
& +\frac{2}{3 \lambda^{2}}\left[\int_{0}^{r} G_{i}(r) d r-\frac{1}{r^{3}} \int_{0}^{r} r^{3} G_{i}(r) d r\right] \\
& +\frac{2}{\lambda^{5} r^{3}}\left[\gamma_{\infty}^{r}(\lambda r) \int_{0}^{r} \delta(\lambda r) G_{i}(r) d r\right. \\
& \left.-\frac{\delta(\lambda r)}{15} \int_{0}^{r} \gamma(\lambda r) G_{i}(r) d r\right]_{\infty}^{r} \text { if } r<a, \\
& -\frac{1}{3 r} \int_{\infty}^{r} r_{i}^{3} G_{i}(r) d r+\frac{1}{3} \int^{2} G_{i}(r) d r \\
F_{i}(r)= & C_{i 3}+C_{i 4}\left(\frac{a}{r}\right)^{3}+\frac{1}{15 r^{3}} \int_{\infty}^{r} r^{5} G_{i}(r) d r
\end{aligned}
$$

for $i=1$ and 2, where

$$
\begin{aligned}
C_{i 1}= & A\left\{a^{2}[\lambda a \cosh (\lambda a)+\sinh (\lambda a)] \int_{\infty}^{a} G_{i}(r) d r\right. \\
& +\gamma(\lambda a) \int_{\infty}^{a} r^{2} G_{i}(r) d r+\frac{2}{\lambda^{2}} \int_{0}^{a} \gamma(\lambda r) G_{i}(r) d r
\end{aligned}
$$

$$
\begin{aligned}
& \left.-\frac{2 \lambda}{3} \cosh (\lambda a) \int_{0}^{a} r^{3} G_{i}(r) d r\right\} \\
& -\frac{2}{3 \lambda^{2}} \int_{0}^{a} G_{i}(r) d r \\
& C_{i 2}=2 A\left\{\int_{\infty}^{a} r^{2} G_{i}(r) d r+\frac{1}{\lambda^{5} a^{3}}\left[2(\lambda a)^{3} \sinh (\lambda a)\right.\right. \\
& +3 \delta(\lambda a)] \int_{0}^{a} \gamma(\lambda r) G_{i}(r) d r \\
& \left.-\left(a^{2}+\frac{3}{\lambda^{2}}\right) \int_{\infty}^{a} G_{i}(r) d r+\frac{1}{\lambda^{2} a^{3}} \int_{0}^{a} r^{3} G_{i}(r) d r\right\} \\
& -\frac{2}{\lambda^{5} a^{3}} \int_{0}^{a} \delta(\lambda r) G_{i}(r) d r \\
& C_{i 3}=A\left\{\frac{a^{2} B}{3} \int_{\infty}^{a} G_{i}(r) d r-\gamma(\lambda a)\left[(\lambda a)^{2} \int_{\infty}^{a} r^{2} G_{i}(r) d r\right.\right. \\
& \left.\left.+\frac{1}{a} \int_{0}^{a} r^{3} G_{i}(r) d r\right]-2 a^{2} \int_{0}^{a} \gamma(\lambda r) G_{i}(r) d r\right\} \\
& +\frac{1}{3 a} \int_{\infty}^{a} r^{3} G_{i}(r) d r \\
& C_{i 4}=A\left\{\frac{B}{3} \int_{\infty}^{a} r^{2} G_{i}(r) d r-\frac{1}{5 \lambda^{2}}\left[\left(\lambda^{2} a^{2}+5\right) B\right.\right. \\
& \left.+3(\lambda a)^{2} \gamma(\lambda a)-2(\lambda a)^{4} \sinh (\lambda a)\right] \int_{\infty}^{a} G_{i}(r) d r \\
& +2\left(a^{2}+\frac{3}{\lambda^{2}}\right) \int_{0}^{a} \gamma(\lambda r) G_{i}(r) d r \\
& \left.-\frac{1}{a}[\lambda a \cosh (\lambda a)+\sinh (\lambda a)] \int_{0}^{a} r^{3} G_{i}(r) d r\right\} \\
& -\frac{1}{15 a^{3}} \int_{\infty}^{a} r^{5} G_{i}(r) d r \\
& G_{1}(r)=\frac{\varepsilon \kappa^{2} a^{2}}{4 \pi Z e r} F_{\mu 0}(r) \frac{d \psi_{\mathrm{eq} 1}}{d r} \\
& G_{2}(r)=-\frac{\varepsilon \kappa^{2} a^{2}}{4 \pi Z e r}\left[F_{\mu 1}(r)+\frac{Z e}{k T} \psi_{\mathrm{eq} 1}(r) F_{\mu 0}(r)\right] \frac{d \psi_{\mathrm{eq} 1}}{d r}, \\
& \gamma(x)=x \cosh x-\sinh x, \\
& \delta(x)=x \sinh x-\cosh x,
\end{aligned}
$$


$A=\left\{\left[3 \lambda a+2(\lambda a)^{3}\right] \cosh (\lambda a)-3 \sinh (\lambda a)\right\}^{-1}$,

$B=\left[6 \lambda a+(\lambda a)^{3}\right] \cosh (\lambda a)-3\left[2+(\lambda a)^{2}\right] \sinh (\lambda a)$.

In Eqs. (14) and (15),

$$
\begin{aligned}
F_{\mu 0}(r)= & \frac{r}{a}+\frac{1-\omega}{2+\omega} \frac{a^{2}}{r^{2}} \quad \text { if } r>a, \\
F_{\mu 0}(r)= & \frac{3}{2+\omega} \frac{r}{a} \quad \text { if } r<a \\
F_{\psi 0}(r)= & \frac{r}{a}+\frac{1-\omega}{2+\omega} \frac{a^{2}}{r^{2}} \\
& -3 \frac{1-\omega}{2+\omega} \frac{a^{2}}{r^{2}}(1+\kappa r) e^{-\kappa(r-a)} \gamma(\kappa a) \\
& \times\left\{(\kappa a)^{3} \cosh (\kappa a)+\left[(\kappa a)^{3}-2 \kappa a-2\right] \sinh (\kappa a)\right. \\
& +2(1+\kappa a) \sinh (\kappa r)\}^{-1} \quad \text { if } r>a
\end{aligned}
$$

$$
\begin{aligned}
F_{\psi 0}(r)= & \frac{3}{2+\omega} \frac{r}{a} \\
& -3 \frac{1-\omega}{2+\omega} \frac{a^{2}}{r^{2}} \gamma(\kappa r)(1+\kappa a)\left\{(\kappa a)^{3} \cosh (\kappa a)\right. \\
& +\left[(\kappa a)^{3}-2 \kappa a-2\right] \sinh (\kappa a) \\
& +2(1+\kappa a) \sinh (\kappa r)\}^{-1} \quad \text { if } r<a
\end{aligned}
$$

$$
\begin{aligned}
F_{\mu 1}(r)= & \frac{1}{2+\omega} \frac{Z e}{k T}\left\{\frac{r}{a} \int_{r}^{a} \frac{d \psi_{\mathrm{eq} 1}}{d r} d r\right. \\
& +\left(\frac{a}{r}\right)^{2} \int_{0}^{r}\left(\frac{r}{a}\right)^{3} \frac{d \psi_{\mathrm{eq} 1}}{d r} d r \\
& -\frac{r}{a} \int_{\infty}^{a}\left[1-2 \frac{1-\omega}{2+\omega}\left(\frac{a}{r}\right)^{3}\right] \frac{d \psi_{\mathrm{eq} 1}}{d r} d r \\
& \left.-2 \frac{1-\omega}{2+\omega} \int_{0}^{a}\left(\frac{r}{a}\right)^{3} \frac{d \psi_{\mathrm{eq} 1}}{d r} d r\right\} \quad \text { if } r<a,
\end{aligned}
$$$$
F_{\mu 1}(r)=\frac{Z e}{3 k T}\left\{-\frac{r}{a} \int_{\infty}^{r}\left[1-2 \frac{1-\omega}{2+\omega}\left(\frac{a}{r}\right)^{3}\right] \frac{d \psi_{\mathrm{eq} 1}}{d r} d r\right.
$$$$
+\left(\frac{a}{r}\right)^{2} \int_{a}^{r}\left(\frac{r}{a}\right)^{3}\left[1-2 \frac{1-\omega}{2+\omega}\left(\frac{a}{r}\right)^{3}\right] \frac{d \psi_{\mathrm{eq} 1}}{d r} d r
$$$$
-\left(\frac{a}{r}\right)^{2} \frac{1-\omega}{2+\omega} \int_{\infty}^{a}\left[1-2 \frac{1-\omega}{2+\omega}\left(\frac{a}{r}\right)^{3}\right] \frac{d \psi_{\mathrm{eq} 1}}{d r} d r
$$$$
\left.+\frac{9 \omega}{(2+\omega)^{2}}\left(\frac{a}{r}\right)^{2} \int_{0}^{a}\left(\frac{r}{a}\right)^{3} \frac{d \psi_{\mathrm{eq} 1}}{d r} d r\right\}
$$

if $r>a$;

$$
\begin{aligned}
F_{\psi 1}(r)= & -\frac{1}{\kappa r^{2}}\left\{( \kappa r + 1 ) e ^ { - \kappa r } \int _ { 0 } ^ { r } \gamma ( \kappa r ) \left[F_{\mu 1}(r)\right.\right. \\
& \left.+\frac{Z e}{k T} \psi_{\mathrm{eq} 1}(r) F_{\mu 0}(r)\right] d r \\
& +\gamma(\kappa r) \int_{r}^{\infty}(\kappa r+1) e^{-\kappa r}\left[F_{\mu 1}(r)\right. \\
& \left.\left.+\frac{Z e}{k T} \psi_{\mathrm{eq} 1}(r) F_{\mu 0}(r)\right] d r\right\}
\end{aligned}
$$

\section{References}

[1] S.S. Dukhin, B.V. Derjaguin, in: E. Matijevic (Ed.), Surface and Colloid Science, Vol. 7, Wiley, New York, 1974.

[2] D.C. Prieve, J.L. Anderson, J.P. Ebel, M.E. Lowell, J. Fluid Mech. 148 (1984) 247.

[3] J.L. Anderson, D.C. Prieve, Langmuir 7 (1991) 403.

[4] J.P. Ebel, J.L. Anderson, D.C. Prieve, Langmuir 4 (1988) 396.

[5] P.O. Staffeld, J.A. Quinn, J. Colloid Interface Sci. 130 (1989) 88.

[6] R.E. Smith, D.C. Prieve, Chem. Eng. Sci. 37 (1982) 1213.

[7] D.C. Prieve, Adv. Colloid Interface Sci. 16 (1982) 321.

[8] D.C. Prieve, R. Roman, J. Chem. Soc. Faraday Trans. 283 (1987) 1287.

[9] Y. Pawar, Y.E. Solomentsev, J.L. Anderson, J. Colloid Interface Sci. 155 (1993) 488.

[10] H.J. Keh, S.B. Chen, Langmuir 9 (1993) 1142.

[11] H.J. Keh, Y.K. Wei, Langmuir 16 (2000) 5289.

[12] H.J. Keh, Y.K. Wei, Langmuir 18 (2002) 10,475.

[13] J.J. Hermans, H. Fujita, K. Nederl. Akad. Wet. Proc. Ser. B 58 (1955) 182.

[14] J.J. Hermans, J. Polym. Sci. 18 (1955) 527.

[15] H.C. Brinkman, Appl. Sci. Res. A 1 (1947) 27.

[16] P. Debye, M. Bueche, J. Chem. Phys. 16 (1948) 573.

[17] N. Imai, K. Iwasa, Israel J. Chem. 11 (1973) 223.

[18] I. Noda, M. Nagasawa, M. Ota, J. Am. Chem. Soc. 86 (1964) 5075.

[19] H. Ohshima, J. Colloid Interface Sci. 163 (1994) 474.

[20] H.J. Keh, Y.C. Liu, J. Colloid Interface Sci. 195 (1997) 169.

[21] Y.C. Liu, H.J. Keh, Langmuir 14 (1998) 1560.

[22] H. Ohshima, T.W. Healy, L.R. White, J. Chem. Soc. Faraday Trans. 279 (1983) 1613.

[23] J. Koplik, H. Levine, A. Zee, Phys. Fluids 26 (1983) 2864.

[24] G.S. Manning, J. Chem. Phys. 51 (1969) 934.

[25] W. Hyk, M. Ciszkowska, J. Phys. Chem. B 103 (1999) 6466.

[26] K. Matsumoto, A. Suganuma, Chem. Eng. Sci. 32 (1977) 445.

[27] J.H. Masliyah, M. Polikar, Can. J. Chem. Eng. 58 (1980) 299.

[28] S. Kawahata, H. Ohshima, N. Muramatsu, T. Kondo, J. Colloid Interface Sci. 138 (1990) 182.

[29] K. Morita, N. Muramatsu, H. Ohshima, T. Kondo, J. Colloid Interface Sci. 147 (1991) 457.

[30] O. Aoyanagi, N. Muramatsu, H. Ohshima, T. Kondo, J. Colloid Interface Sci. 162 (1994) 222.

[31] V.G. Levich, Physicochemical Hydrodynamics, Prentice-Hall, Englewood Cliffs, NJ, 1962

[32] G. Neale, N. Epstein, W. Nader, Chem. Eng. Sci. 28 (1973) 1865.

[33] V. Natraj, S.B. Chen, J. Colloid Interface Sci. 251 (2002) 200.

[34] D.C. Henry, Proc. R. Soc. London Ser. A 133 (1931) 106.

[35] R.W. O'Brien, L.R. White, J. Chem. Soc. Faraday Trans. 274 (1978) 1607. 